\title{
Conocimientos y creencias acerca de epilepsia en los padres de familia usuarios habituales del centro de salud de Peralvillo - Huaral.
}

\author{
Knowledge and beliefs about epilepsy among parents who are frequent users of a Health Center in Peralvillo \\ - Huaral.
}

Rosselym S. Giraldo-Tapia ${ }^{1,2}$, Melina Tejero-Alcalde ${ }^{1,2}$, Julio E. Urdániga-Alvarado ${ }^{1,2}$, Frine Samalvides-Cuba ${ }^{2,5,6}$, Daniel Guillén-Pinto 2,3,4

\section{RESUMEN}

La epilepsia es una enfermedad crónica frecuente en niños, poco conocida y habitualmente estigmatizada. En los países en desarrollo es más frecuente en las áreas rurales y generalmente es de causa secundaria. Objetivos: Determinar el nivel de conocimiento y creencias sobre epilepsia en los padres de familia usuarios habituales del Centro de Salud de Peralvillo - Huaral. Materiales y Métodos: Estudio descriptivo y transversal. Se aplicó un instrumento anónimo validado para determinar el nivel de conocimiento y creencias en una población de padres de familia del Asentamiento Humano Peralvillo Huaral, Lima. Resultados: Se realizaron 104 entrevistas. El conocimiento nulo se encontró en el 44,2\%. El 31\% tuvo por lo menos una creencia siendo la más frecuente que el paciente en estado de crisis puede volverse agresivo. Se encontró una relación directa entre grado de instrucción y el nivel de conocimiento, no se observó asociación entre las demás variables sociodemográficas y el conocimiento. Conclusiones: En la población investigada se encontró bajo nivel de conocimiento sobre epilepsia en niños. El grado de instrucción, a diferencia de las demás características sociodemográficas, mostró una relación directa con nivel de conocimiento. Fueron frecuentes las creencias.

PALABRAS CLAVES: Epilepsia, conocimiento, creencias, niños, crisis, Huaral

\section{SUMMARY}

Epilepsy is a chronic common disease in children, little known and commonly stigmatized. In developing countries is more common in rural areas and generally by secondary cause.

Objectives: To determine the level of knowledge and beliefs about epilepsy in parents of the Health Micro-Net of Peralvillo-Huaral. Materials and Methods: Descriptive transversal study. A validated anonymous quiz was applied to a group of parents of Peralvillo-Huaral health center, to determinate knowledge and beliefs level about epilepsy in children. Results: One hundred and four interviews were performed, $44.2 \%(46)$ had no knowledge. $31 \%$ had at least one belief; the most frequent was to consider that an epileptic patient can be aggressive during a seizure. We found a direct relationship between level of education and the level of knowledge, there was no association between

1. Médico Egresado

2. Facultad de Medicina Alberto Hurtado. Universidad Peruana Cayetano Heredia. Lima, Perú

3. Neurólogo Pediatra, Profesor Principal.

4. Jefe del Servicio de Especialidades Pediátricas, Hospital Nacional Cayetano Heredia.

5. Magíster en Epidemiología Clínica. Instituto de Medicina Tropical Alexander von Humboldt Hospital Nacional Cayetano Heredia, Lima Perú.

6. Médico Asistente. Departamento de Enfermedades Infecciosas, Tropicales y Dermatológicas. Hospital Nacional Cayetano Heredia. Lima, Perú. 
Conocimientos y creencias acerca de epilepsia en los padres de familia usuarios habituales del centro de salud.

socio-demographic and other knowledge. Conclusions: A low knowledge level about epilepsy in children was found in the studied population. Level of education, unlike the other social demographic characters, showed a direct relationship with knowledge level. Beliefs were frequent.

KEY WORDS: Epilepsy, knowledge, beliefs, seizure, children, Huaral.

\section{INTRODUCCIÓN}

La Epilepsia es una enfermedad caracterizada por la predisposición permanente para generar crisis epilépticas (1). Las crisis son síntomas de disfunción cerebral causadas por una descarga hipersincrónica, paroxística y autolimitada de un grupo de neuronas hiperexcitables. Sus manifestaciones clínicas son muy variables, de acuerdo a su origen pueden ser consecuencia de una disfunción cerebral transitoria, lesiones permanentes o formar parte de un síndrome epiléptico específico (2). Las personas que padecen de epilepsia tienen un riesgo de morir 2 a 4 veces más elevado que la población general. Aproximadamente más de dos tercios de los pacientes tratados, pueden presentar remisión total (1).

En los países desarrollados la epilepsia tiene una prevalencia de 3-4/1000 habitantes, mientras que en los países en desarrollo varía entre 5-77/1000 habitantes (2), en América Latina su prevalencia de epilepsia activa es de 12.4/1000, en estos países gran parte de los pacientes se encuentran en el sector rural y generalmente tienen epilepsia de causa secundaria, potencialmente prevenible. (3)

En los estudio de Jarvie y col. en 1993 y de Shaw y col. en el 2008 se menciona que es muy importante que el paciente conozca sobre su enfermedad, en estos estudios se determinó que el cuestionario validado puede ser una herramienta útil para la evaluación del conocimiento; y finalmente los programas de educación de autocuidado para personas con epilepsia, usando métodos psicosociales, pueden mejorar el conocimiento sobre la epilepsia, y reducir la frecuencia de crisis epilépticas $(4,5)$.

En el estudio transversal realizado por Espinoza y col en el 2002, en el sur de Lima, se compararon resultados de un cuestionario aplicado a un grupo de profesores en el año 1992 y nuevamente aplicado en el 2000 , con el fin de conocer sus actitudes, conocimientos y creencias sobre la epilepsia, antes y después de una intervención educativa. Dicha intervención consistió en brindar programas educativos a los profesores a lo largo de ocho años. Al final del estudio se concluyó que los prejuicios y creencias se mantenían, sin embargo la información recibida permitió a la mayoría de profesores saber qué hacer ante una crisis, y aumentó el porcentaje que consideraban que el rendimiento escolar y la capacidad de aprender son iguales en los niños con y sin epilepsia (6).

En otro estudio realizado por González y col. acerca de actitudes y creencias en educadores de círculos infantiles sobre epilepsia, se menciona que los factores mágico-religiosos ligados a los antecedentes de esta enfermedad han condicionado el rechazo social de las personas afectadas. Además, concluyen que el conocimiento de estas educadoras acerca de la epilepsia es pobre, sin saber la conducta que se debe seguir ante una crisis epiléptica y que dicho conocimiento fue adquirido sin fundamento científico (7).

Es importante realizar este estudio porque actualmente no existe información acerca de cuál es el nivel de conocimiento de una población rural con respecto a epilepsia en la infancia que permita realizar intervenciones educativas con la finalidad de difundir el concepto de la enfermedad, la atención de las crisis y la importancia de la adherencia al tratamiento.

El presente es un proyecto de investigación que tiene la finalidad de evaluar el nivel de conocimiento sobre epilepsia en una población rural usando un instrumento validado. El cual es aplicable a un grupo focalizado, en una población que no está distribuida aleatoriamente. Este cuestionario es aplicable en la comunidad seleccionada, "Comité 16", porque se incluirán padres de familia usuarios habituales del centro de salud, quienes reflejarán en gran medida el trabajo preventivo-promocional realizado en cuanto a epilepsia así como aquellos aspectos en los que aún es necesario trabajar.

El objetivo de este estudio fue describir los conocimientos y creencias acerca de Epilepsia en los Padres de Familia, usuarios habituales del Centro de Salud de Peralvillo - Huaral.

Asimismo, explorar las correlaciones de edad, grado de instrucción, procedencia y número de hijos con nivel de conocimientos y creencias de los jefes de familia. 


\section{MATERIAL Y MÉTODOS}

Estudio descriptivo de corte transversal tipo encuesta de salud realizado en el Asentamiento Humano Comité 16 del centro poblado de Peralvillo de la provincia de Huaral, Lima, donde se aplicó un cuestionario validado (8) con visitas domiciliarias, previa autorización de los representantes de la comunidad y en conocimiento de las autoridades del Centro de Salud de Peralvillo. La información recolectada fue anónima y se mantuvieron los datos en reserva de los investigadores.

Para garantizar la calidad metodológica del estudio se realizó un muestreo no probabilístico consecutivo, en el que se incluyó a todos los padres de familia usuarios habituales del Centro de Salud de Peralvillo en Huaral, pertenecientes al Asentamiento Humano Comité 16. La población determinada fue de 104 familias, considerando que hay un registro de 149 viviendas, de las cuales aproximadamente 45 se encontraron deshabitadas.

Dentro de los criterios de inclusión se consideraron: ser padre de familia usuario habitual del Centro de Salud de Peralvillo y poblador residente del Comité 16 de Peralvillo. Los criterios de exclusión fueron: tener algún trastorno o enfermedad psiquiátrica $\mathrm{o}$ neurológica con discapacidad mayor, que impida el llenado del cuestionario, ser padre de familia iletrado, ser padre de familia con epilepsia y ser padre de familia de un hijo con epilepsia.

Las variables sociodemográficas edad, sexo, grado de instrucción, lugar de nacimiento, tiempo de residencia y número de hijos se cruzaron contra el nivel de conocimiento, y la frecuencia de creencias. Para analizar el nivel de conocimiento se utilizó el instrumento validado categorizándolo como bajo cuando el puntaje obtenido por el padre de familia se encontraba desde 1 a 22, intermedio cuando el puntaje era de 23 a 29, y alto cuando el puntaje era mayor de 29. A aquellos padres que respondieron no "saber algo acerca de epilepsia" se les asignó un puntaje de cero considerándolos como grupo con conocimiento nulo. (Anexo 1). En cuanto a las variables de creencias, se determinó su frecuencia mediante el cuestionario.

Las variables cuantitativas número de hijos $\mathrm{y}$ tiempo de residencia se categorizaron en función a sus medias, mientras que la variable edad se estratificó según la Organización Mundial de la Salud. Para el análisis del grado de instrucción, debido a su tendencia, se agruparon las categorías secundaria y superior, mientras que la categoría primaria permaneció como tal.

Una vez recolectada la información fue introducida a una base de datos diseñada específicamente para este estudio en una hoja de cálculo del programa Excel y luego fue analizada mediante el programa SPSS v15.0.

El estudio fue previamente autorizado por el comité de ética de la Universidad Peruana Cayetano Heredia quien supervisó que no existiera conflicto ético durante su ejecución.

\section{RESULTADOS}

Se realizaron 104 entrevistas a padres de familia residentes del Asentamiento Humano Comité 16 perteneciente al centro poblado de Peralvillo, durante el mes de febrero del 2013.

De la muestra con la que se trabajó, el $49 \%$ tuvieron una edad comprendida entre 35-60 años, las mujeres representaron el 87,5\%. El 28,8\% de los entrevistados nacieron en el distrito de Chancay, 31,7 \% procedían de Ancash y el 39,5\% de otros departamentos del interior del país. El 41,3\% residen más 20 años, el $61,5 \%$ tienen 3 o más hijos. El grado de instrucción fue predominantemente nivel de secundaria, $55,8 \% \mathrm{y}$ nivel primaria con $36,5 \%$ (Tabla 1 ).

El conocimiento nulo correspondió al 44,2\%, el nivel intermedio $33,7 \%$, el nivel bajo $15,4 \%$ y el nivel alto $6,7 \%$ (Gráfico1).

Sólo se evaluaron las creencias en el grupo que contestó tener algún conocimiento acerca de Epilepsia, el 32,8\% de los padres de familia cree que "el paciente con epilepsia puede ser agresivo durante una crisis", $10,3 \%$ cree que "una persona con epilepsia no es útil a su comunidad", $8,6 \%$ cree que "un niño con epilepsia no puede acudir a una escuela regular", 6,9\% cree que "un niño epiléptico tiene un menor nivel de inteligencia en comparación a otro sin la enfermedad" y 1,7\% manifestó la creencia de "no permitir a su hijo tener un amigo epiléptico" (Gráfico 2).

El 56,8\% de los entrevistados, no tiene creencias, $31 \%$ tiene una creencia, $10,3 \%$ tiene dos creencias, menos del $4 \%$ tiene tres y cuatro creencias y ninguno presentó las cinco creencias (Gráfico 3).

Al confrontarse las variables se obtuvo que 
Conocimientos y creencias acerca de epilepsia en los padres de familia usuarios habituales del centro de salud.

Tabla 1. Nivel de Conocimiento en padres de familia usuarios habituales del Centro de Salud Peralvillo-Huaral

\begin{tabular}{|c|c|c|c|c|c|c|}
\hline \multirow{2}{*}{\multicolumn{2}{|c|}{ Variables Sociodemográficas }} & \multicolumn{4}{|c|}{ Nivel de Conocimiento } & \multirow{3}{*}{$\mathbf{P}$} \\
\hline & & \multicolumn{2}{|c|}{ Nulo - Bajo } & \multicolumn{2}{|c|}{ Intermedio - Alto } & \\
\hline & & $\mathbf{n}$ & $\%$ & $\mathbf{n}$ & $\%$ & \\
\hline \multirow{2}{*}{ Sexo } & Femenino & 57 & 62,7 & 34 & 37,4 & \multirow{2}{*}{0.09} \\
\hline & Masculino & 5 & 38,5 & 8 & 61,5 & \\
\hline \multirow{2}{*}{ Edad } & 18 a 35 & 21 & 52,5 & 19 & 47,5 & \multirow{2}{*}{0.24} \\
\hline & $>35$ & 41 & 64,1 & 23 & 35,9 & \\
\hline \multirow{2}{*}{ Número de hijos } & $<3$ & 20 & 50,0 & 20 & 50,0 & \multirow{2}{*}{0.11} \\
\hline & $\geq 3$ & 42 & 65,6 & 22 & 34,4 & \\
\hline \multirow{2}{*}{ Lugar de Nacimiento } & Costa & 32 & 52,2 & 27 & 45,8 & \multirow{2}{*}{0.2} \\
\hline & Sierra / Selva & 30 & 66,7 & 15 & 33,3 & \\
\hline \multirow{2}{*}{ Grado de Instrucción } & Primaria & 30 & 78,9 & 28 & 21,1 & \multirow{2}{*}{0.002} \\
\hline & Secundaria / Superior & 32 & 48,5 & 14 & 51,5 & \\
\hline \multirow{2}{*}{$\begin{array}{l}\text { Tiempo de Residencia } \\
\text { en años }\end{array}$} & $\leq \mathbf{2 0}$ & 39 & 63,9 & 22 & 36,1 & \multirow{2}{*}{0.29} \\
\hline & $>20$ & 23 & 53,5 & 20 & 46,5 & \\
\hline
\end{tabular}

Gráfico 1. Frecuencia de las categorías de conocimiento en los padres de familia usuarios habituales del Centro de Salud Peralvillo-Huaral

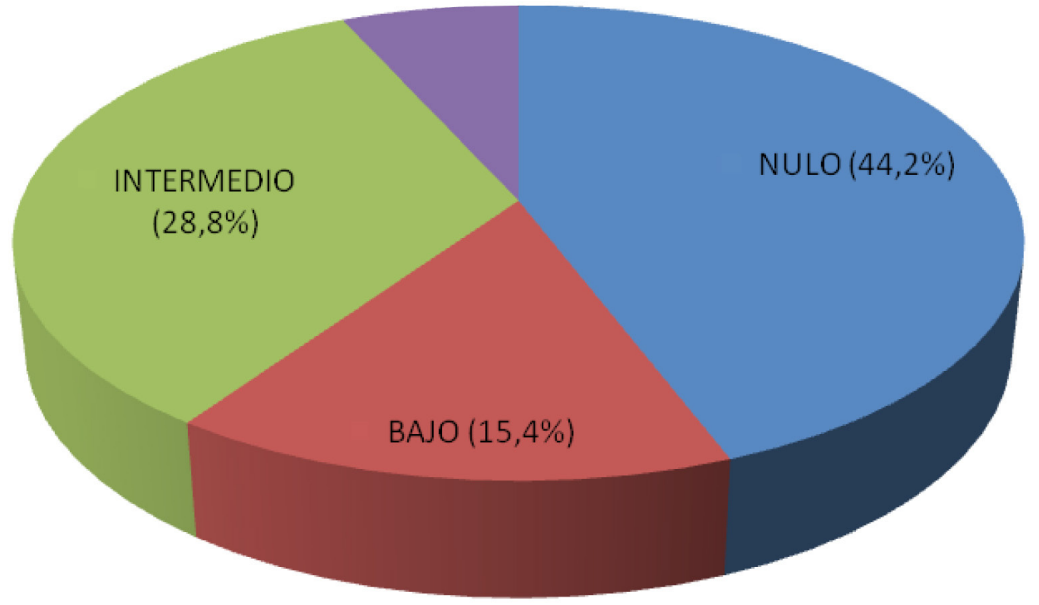

existe relación directa entre el grado de instrucción y conocimiento, así el 78,9\% de los entrevistados con grado de instrucción primaria, tenían un nivel de conocimiento nulo-bajo; en tanto que el 51,5\% de aquellos con grado de instrucción secundaria o superior, obtuvieron un nivel de conocimiento intermedio-alto, con un nivel de significancia $(\mathrm{p}=0,002)$. Incluso, al dicotomizar las categorías del conocimiento ( $\mathrm{Si}$ conoce, No conoce) el $68,4 \%$ de los padres con grado de instrucción primaria respondió no "tener conocimiento acerca de epilepsia", mientras que el $69,7 \%$ de aquellos con grado de instrucción secundaria o superior respondió sí "tener conocimiento acerca de epilepsia", con un mejor nivel de significancia $(\mathrm{p}<0,001)$. Asimismo, no se encontró asociación entre las demás características sociodemográficas y conocimiento.

\section{DISCUSIÓN}

La epilepsia tiene un exagerado impacto negativo en la población relacionado con el bajo nivel de información y desproporcionado con su prevalencia (9). En ese sentido, el conocimiento de la frecuencia, etiología y pronóstico de la epilepsia ha mejorado notablemente gracias a los estudios epidemiológicos 
Giraldo R, et al.

Gráfico 2. Frecuencia del tipo de creencia acerca del niño con epilepsia en padres de familia usuarios habituales del Centro de Salud Peralvillo-Huaral

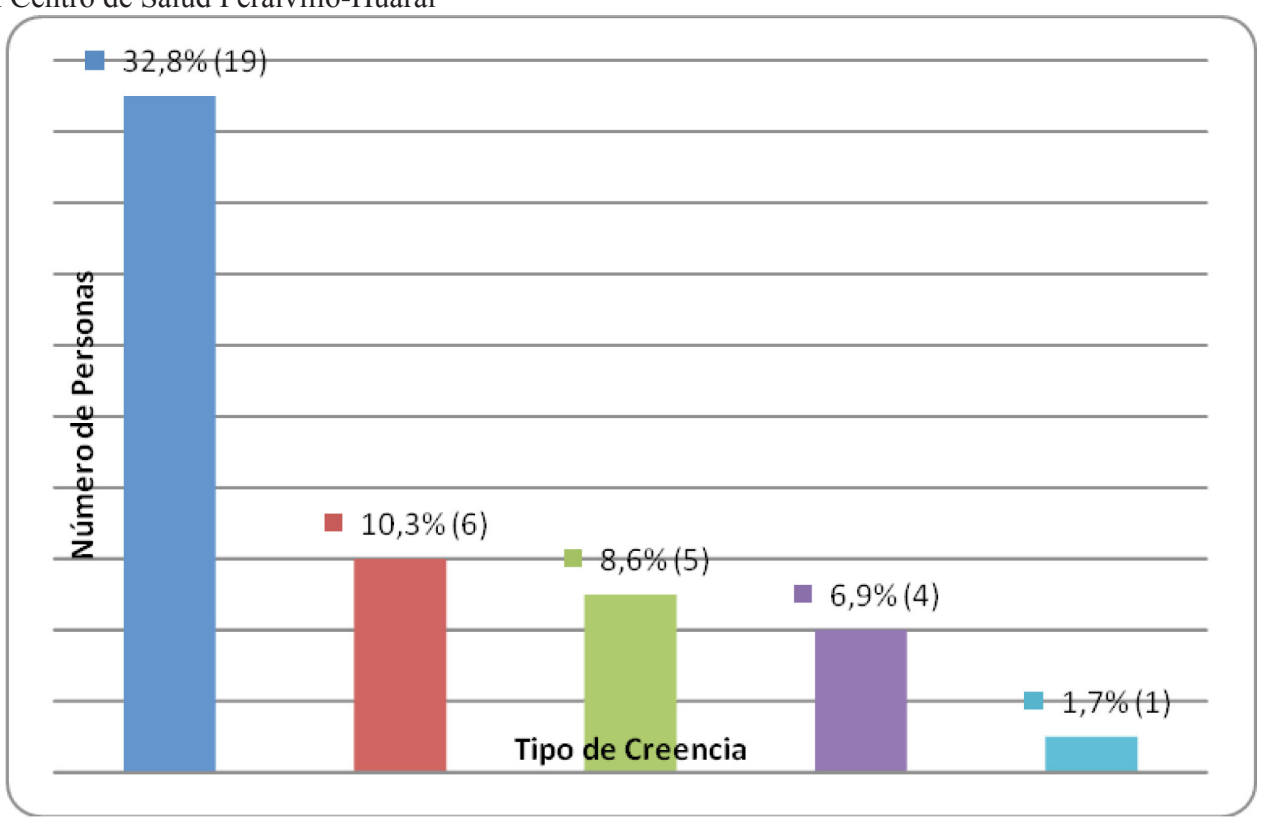

1. Puede volverse agresivo durante una crisis

2. No puede ser útil a su comunidad

3. No puede acudir a la misma escuela que un niño sin epilepsia

4. No permitiría que fuera amigo de su hijo

5. Es menos inteligente que un niño sin epilepsia

Gráfico 3. Frecuencia acumulada de las creencias acerca del niño con epilepsia en padres de familia usuarios habituales del Centro de Salud Peralvillo-Huaral

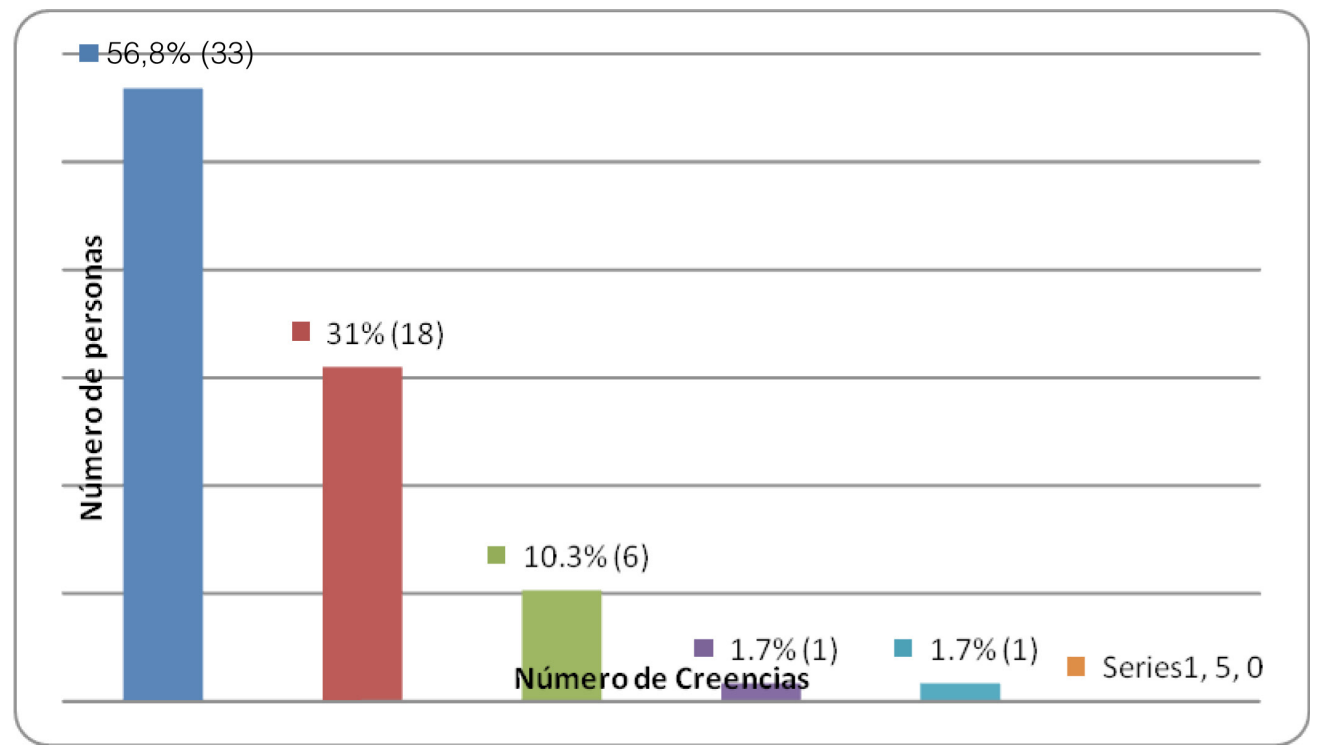


Conocimientos y creencias acerca de epilepsia en los padres de familia usuarios habituales del centro de salud.

Tabla 2. Tabla de Creencias en padres de familia usuarios habituales del Centro de Salud

\begin{tabular}{|c|c|c|c|c|c|c|c|c|c|c|c|}
\hline \multirow{3}{*}{\multicolumn{2}{|c|}{$\begin{array}{c}\text { Variables } \\
\text { Sociodemográficas }\end{array}$}} & \multicolumn{10}{|c|}{ Creencias acerca del niño epiléptico } \\
\hline & & \multicolumn{2}{|c|}{$\begin{array}{l}\text { Puede volverse } \\
\text { agresivo durante } \\
\text { una crisis }\end{array}$} & \multicolumn{2}{|c|}{$\begin{array}{c}\text { Puede acudir a } \\
\text { la misma escuela } \\
\text { que un niño sin } \\
\text { epilepsia }\end{array}$} & \multicolumn{2}{|c|}{$\begin{array}{l}\text { Puede ser útil a } \\
\text { su comunidad }\end{array}$} & \multicolumn{2}{|c|}{$\begin{array}{l}\text { Permitiría que } \\
\text { fuera amigo de } \\
\text { su hijo }\end{array}$} & \multicolumn{2}{|c|}{$\begin{array}{l}\text { Es menos } \\
\text { inteligente que } \\
\text { un niño sin } \\
\text { epilepsia }\end{array}$} \\
\hline & & $\begin{array}{c}\text { Sí } \\
\mathbf{n} \\
(\%)\end{array}$ & $\begin{array}{c}\text { No } \\
\mathbf{n} \\
(\%)\end{array}$ & $\begin{array}{c}\text { Sí } \\
\mathbf{n} \\
(\%)\end{array}$ & $\begin{array}{c}\text { No } \\
\text { n } \\
(\%) \\
\end{array}$ & $\begin{array}{c}\text { Sí } \\
\text { n } \\
(\%)\end{array}$ & $\begin{array}{c}\text { No } \\
\text { n } \\
(\%)\end{array}$ & $\begin{array}{c}\text { Sí } \\
\mathbf{n} \\
(\%)\end{array}$ & $\begin{array}{c}\text { No } \\
\mathbf{n} \\
(\%)\end{array}$ & $\begin{array}{c}\text { Sí } \\
\text { n } \\
(\%)\end{array}$ & $\begin{array}{c}\text { No } \\
\text { n } \\
(\%)\end{array}$ \\
\hline \multirow{2}{*}{ Sexo } & Femenino & $\begin{array}{c}16 \\
(33,3)\end{array}$ & $\begin{array}{c}32 \\
(66,7)\end{array}$ & $\begin{array}{c}43 \\
(89,6)\end{array}$ & $\begin{array}{c}5 \\
(10,4)\end{array}$ & $\begin{array}{c}43 \\
(89,6)\end{array}$ & $\begin{array}{c}5 \\
(10,4)\end{array}$ & $\begin{array}{c}47 \\
(97,9)\end{array}$ & $\begin{array}{c}1 \\
(2,1)\end{array}$ & $\begin{array}{c}3 \\
(6,3)\end{array}$ & $\begin{array}{c}45 \\
(93,8)\end{array}$ \\
\hline & Masculino & $\begin{array}{c}3 \\
(30,0)\end{array}$ & $\begin{array}{c}7 \\
(70,0)\end{array}$ & $\begin{array}{c}10 \\
(100,0)\end{array}$ & $\begin{array}{c}0 \\
(0,0)\end{array}$ & $\begin{array}{c}9 \\
(90,0)\end{array}$ & $\begin{array}{c}1 \\
(10,0)\end{array}$ & $\begin{array}{c}10 \\
(100,0)\end{array}$ & $\begin{array}{c}0 \\
(0,0)\end{array}$ & $\begin{array}{c}1 \\
(10,0)\end{array}$ & $\begin{array}{c}9 \\
(90,0)\end{array}$ \\
\hline \multirow{2}{*}{ Edad (años) } & $\leq 35$ & $\begin{array}{c}9 \\
(36,0)\end{array}$ & $16(64,0)$ & $\begin{array}{c}22 \\
(88,0)\end{array}$ & $\begin{array}{c}3 \\
(12,0)\end{array}$ & $\begin{array}{c}22 \\
(68,0)\end{array}$ & $\begin{array}{c}3 \\
(12,0)\end{array}$ & $\begin{array}{c}24 \\
(96,0)\end{array}$ & $\begin{array}{c}1 \\
(4,0)\end{array}$ & $\begin{array}{c}4 \\
(84,0)\end{array}$ & $\begin{array}{c}21 \\
(16,0)\end{array}$ \\
\hline & $>35$ & $10(30,3)$ & $23(69,7)$ & $\begin{array}{c}31 \\
(93,9)\end{array}$ & $\begin{array}{c}2 \\
(6,1)\end{array}$ & $\begin{array}{c}30 \\
(90,9)\end{array}$ & $\begin{array}{c}3 \\
(9,1)\end{array}$ & $\begin{array}{c}33 \\
(100,0)\end{array}$ & $\begin{array}{c}0 \\
(0,0)\end{array}$ & $\begin{array}{c}0 \\
(0,0)\end{array}$ & $\begin{array}{c}33 \\
(100,0)\end{array}$ \\
\hline \multirow{2}{*}{$\begin{array}{l}\text { Número de } \\
\text { hijos }\end{array}$} & $<3$ & $\begin{array}{c}10 \\
(41,7)\end{array}$ & $\begin{array}{c}14 \\
(58,3)\end{array}$ & $\begin{array}{c}21 \\
(87,5)\end{array}$ & $\begin{array}{c}3 \\
(12,5)\end{array}$ & $\begin{array}{c}21 \\
(87,5)\end{array}$ & $\begin{array}{c}3 \\
(12,5)\end{array}$ & $\begin{array}{c}24 \\
(100,0)\end{array}$ & $\begin{array}{c}0 \\
(0,0)\end{array}$ & $\begin{array}{c}2 \\
(8,3)\end{array}$ & $\begin{array}{c}22 \\
(91,7)\end{array}$ \\
\hline & $\geq 3$ & $\begin{array}{c}9 \\
(26,5)\end{array}$ & $\begin{array}{c}25 \\
(73,5)\end{array}$ & $\begin{array}{c}32 \\
(94,1)\end{array}$ & $\begin{array}{c}2 \\
(5,9)\end{array}$ & $\begin{array}{c}31 \\
(91,2)\end{array}$ & $\begin{array}{c}3 \\
(8,8)\end{array}$ & $\begin{array}{c}33 \\
(97,1)\end{array}$ & $\begin{array}{c}1 \\
(2,9)\end{array}$ & $\begin{array}{c}2 \\
(5,9)\end{array}$ & $\begin{array}{c}32 \\
(94,1)\end{array}$ \\
\hline \multirow{2}{*}{$\begin{array}{c}\text { Lugar de } \\
\text { Nacimiento }\end{array}$} & Costa & $\begin{array}{c}12 \\
(33,3)\end{array}$ & $\begin{array}{c}24 \\
(66,7)\end{array}$ & $\begin{array}{c}33 \\
(91,7)\end{array}$ & $\begin{array}{c}3 \\
(8,3)\end{array}$ & $\begin{array}{c}31 \\
(86,1)\end{array}$ & $\begin{array}{c}5 \\
(13,5)\end{array}$ & $\begin{array}{c}36 \\
(100,0)\end{array}$ & $\begin{array}{c}0 \\
(0,0)\end{array}$ & $\begin{array}{c}2 \\
(5,6)\end{array}$ & $\begin{array}{c}34 \\
(94,4)\end{array}$ \\
\hline & Sierra y Selva & $\begin{array}{c}7 \\
(31,8)\end{array}$ & $\begin{array}{c}15 \\
(68,2)\end{array}$ & $\begin{array}{c}20 \\
(91,3)\end{array}$ & $\begin{array}{c}2 \\
(8,7)\end{array}$ & $\begin{array}{c}21 \\
(95,5)\end{array}$ & $\begin{array}{c}1 \\
(4,5)\end{array}$ & $\begin{array}{c}21 \\
(95,5)\end{array}$ & $\begin{array}{c}1 \\
(4,5)\end{array}$ & $\begin{array}{c}2 \\
(9,1)\end{array}$ & $\begin{array}{c}20 \\
(90,9)\end{array}$ \\
\hline \multirow{2}{*}{$\begin{array}{l}\text { Grado de } \\
\text { Instrucción }\end{array}$} & Primaria & $\begin{array}{c}8 \\
(33,3)\end{array}$ & $\begin{array}{c}4 \\
(66,7)\end{array}$ & $\begin{array}{c}11 \\
(91,7)\end{array}$ & $\begin{array}{c}1 \\
(8,3)\end{array}$ & $\begin{array}{c}9 \\
(75,0)\end{array}$ & $\begin{array}{c}3 \\
(25,0)\end{array}$ & $\begin{array}{c}11 \\
(91,7)\end{array}$ & $\begin{array}{c}1 \\
(8,3)\end{array}$ & $\begin{array}{c}2 \\
(16,7)\end{array}$ & $\begin{array}{c}10 \\
(83,3)\end{array}$ \\
\hline & $\begin{array}{c}\text { Secundaria y } \\
\text { Superior }\end{array}$ & $\begin{array}{c}15 \\
(32,6)\end{array}$ & $\begin{array}{c}31 \\
(66,4)\end{array}$ & $\begin{array}{c}42 \\
(90,9)\end{array}$ & $\begin{array}{c}4 \\
(9,1)\end{array}$ & $\begin{array}{c}43 \\
(93,5)\end{array}$ & $\begin{array}{c}3 \\
(6,5)\end{array}$ & $\begin{array}{c}46 \\
(100,0)\end{array}$ & $\begin{array}{c}0 \\
(0,0)\end{array}$ & $\begin{array}{c}2 \\
(4,3)\end{array}$ & $\begin{array}{c}44 \\
(95,7)\end{array}$ \\
\hline \multirow{2}{*}{$\begin{array}{l}\text { Tiempo de } \\
\text { Residencia } \\
\text { (años) }\end{array}$} & $\leq 20$ & $\begin{array}{c}10 \\
(29,4)\end{array}$ & $\begin{array}{c}24 \\
(70,6)\end{array}$ & $\begin{array}{c}32 \\
(94,1)\end{array}$ & $\begin{array}{c}2 \\
(5,9)\end{array}$ & $\begin{array}{c}31 \\
(91,2)\end{array}$ & $\begin{array}{c}3 \\
(8,8)\end{array}$ & $\begin{array}{c}33 \\
(97,1)\end{array}$ & $\begin{array}{c}1 \\
(2,9)\end{array}$ & $\begin{array}{c}4 \\
(8,8)\end{array}$ & $\begin{array}{c}21 \\
(91,2)\end{array}$ \\
\hline & $>\mathbf{2 0}$ & $\begin{array}{c}9 \\
(37,5)\end{array}$ & $\begin{array}{c}15 \\
(62,5)\end{array}$ & $\begin{array}{c}21 \\
(87,5)\end{array}$ & $\begin{array}{c}3 \\
(12,5)\end{array}$ & $\begin{array}{c}21 \\
(87,5)\end{array}$ & $\begin{array}{c}3 \\
(12,5)\end{array}$ & $\begin{array}{c}24 \\
(100,0)\end{array}$ & $\begin{array}{c}0 \\
(0,0)\end{array}$ & $\begin{array}{c}0 \\
(4,2)\end{array}$ & $\begin{array}{c}33 \\
(95,8)\end{array}$ \\
\hline
\end{tabular}

realizados a lo largo de todo el mundo, algunos de los cuales mencionan que el conocimiento de la enfermedad tiene una influencia favorable en la calidad de vida de los pacientes epilépticos $(1,7)$. Por ejemplo, en el estudio de Jarvie y col. en 1993, se concluyó que el adecuado nivel de conocimiento en pacientes con epilepsia es un factor fundamental para enfrentar con éxito el tratamiento de su enfermedad (5).

A nivel comunitario, esto significa que el personal de los establecimientos de salud tiene un importante rol en la difusión de información y educación a la población, herramienta fundamental para identificar oportunamente los casos probables, contribuir al conocimiento de la prevención y fortalecer la adherencia al tratamiento de la epilepsia infantil.

Las investigaciones han demostrado que la epilepsia es más frecuente en las zonas rurales, en donde los pacientes sólo tienen acceso al nivel primario de atención en salud. En una revisión sistemática realizada por Meyer AC y col. en el 2010 se concluyó que la epilepsia puede ser manejada por médicos de primer nivel de atención en salud usando medicamentos de menor costo e igual eficacia (primera línea de tratamiento) (10).

La población investigada se caracterizó por estar conformada por amas de casa, en su mayoría de 35 a 60 años de edad, provenientes del distrito aledaño de Chancay y de la región Ancash, la mitad de ellas fueron multíparas, con grado de instrucción secundaria y asentadas gran parte de su vida en ésta comunidad.

A pesar de esta homogeneidad poblacional fue interesante observar que sólo el grado de instrucción tuvo relación directa con el nivel conocimiento en epilepsia.

En general, los resultados indican que ésta población tiene bajo nivel de conocimiento sobre epilepsia en la infancia, 44,2\% puede ser sorprendente, pero sólo 
futuros estudios determinaran que tan bajo nivel tiene con respecto a otras similares. en un estudio de corte transversal realizado por Saengsuwan $\mathrm{J}$ en el año 2012 , se demuestra que el $50 \%$ de los pacientes con epilepsia en Tailandia, creen que su enfermedad es un desorden mental o enfermedad psiquiátrica, por tanto es deseable que la población este mejor informada, Sin embargo son escasas las referencias sobre las condiciones ideales de información y nivel cultural de salud (11).

Son varias las hipótesis para explicar estos hallazgos, pero la más probable es que la población desconoce el tema porque la información es insuficiente, porque no se realizan campañas salud, no se difunde habitualmente estos conceptos en los centros educativos, en centros de salud y en los medios de difusión social, también es probable que el perfil de la población entrevistada, que fueron mujeres mayoritariamente amas de casa y madres con gran carga familiar, no tengan acceso, tiempo y facilidades para conseguir la información, excepto por las vivencias.

Con respecto a las creencias, obtuvimos resultados similares a estudios previos, por ejemplo, el estudio realizado por J. Espinoza y col. en 2002 reveló la persistencia significativa de ideas erróneas y prejuicios acerca de los niños con epilepsia como el de no "ser capaz de culminar sus estudios con éxito" (6).

Este hallazgo es muy importante porque pone en evidencia una realidad que cotidianamente se observa en la práctica clínica y que hace dudar a los padres de familia sobre la utilidad de la medicación.ç

Cerca del $50 \%$ de los padres de familia que respondieron tener "algún nivel de conocimiento", no presentaron creencias; asimismo, en el grupo que sí las tuvo, fue más frecuente una sola creencia, siendo ésta la de considerar que el paciente en estado de crisis puede volverse agresivo y la segunda, que el niño epiléptico no puede acudir a una escuela regular. Una posible explicación sería la experiencia adquirida a través de vivencias con personas con epilepsia, la cual les ayudó a disminuir algunas ideas basadas en pensamientos místicos acerca de la enfermedad (6).

La agresividad durante una crisis es la creencia más frecuente, es probable que la persistencia de concepciones mágico-religiosas como la "posesión maligna" entre otras estén vinculadas al desarrollo de la crisis en la cultura de la población estudiada.
Las siguientes creencias frecuentes fueron "el niño epiléptico no es útil a su comunidad" y "el niño epiléptico no puede acudir a la misma escuela que un niño sin epilepsia", pueden significar que muchos padres de familia piensan que la epilepsia es una enfermedad que invalida al niño volviéndolo incapaz de culminar sus estudios con éxito y por lo tanto no realizar un aporte en beneficio de su comunidad $(5,7)$. Se encontró una relación directa entre grado de instrucción y nivel de conocimiento, que por razones lógicas era de esperar, sin embargo este conocimiento muchas veces puede ser reforzada e interiorizada por experiencias con familiares, vecinos o amigos con epilepsia. Sin embargo, aunque a veces se aprende que hacer ante emergencias, es posible que la creencia no se haya corregido como lo demostró el estudio de Espinoza y col. en 2002 quienes concluyeron que un grupo de profesores tenían ideas equivocadas y prejuicios respecto a niños con epilepsia, a pesar de un programa de información mensual durante ocho años (6).

Al comparar las demás características sociodemográficas (edad, sexo, número de hijos, lugar de nacimiento y tiempo de residencia) con el conocimiento, no se halló diferencia significativa entre el grupo con algún nivel de conocimiento y el que presenta un conocimiento nulo, tampoco se encontró diferencia al comparar las categorías de conocimiento nulo-bajo con intermedio-alto. Como se señala en el estudio de Tardillo y col, en el 2009, en Peralvillo, sobre conocimientos en meningitis, también con población mayoritariamente femenina, no se encontró relación entre nivel de conocimiento sobre meningitis y las variables sociodemográficas (12). Aun así, es probable que nuestros hallazgos estén sesgados por el tamaño y selección de la población.

Las limitaciones de este estudio fueron la participación escasa de varones en las entrevistas domiciliarias, quienes en su mayoría se encontraban laborando, lo cual no permite demostrar la predominancia de género. Asimismo, el instrumento no identifica las denominaciones alternativas sobre epilepsia ("ataques", "tembladera", "nervios") y esto pudo reducir el número de participantes evaluables para las creencias. La tercera limitación de este estudio consiste en la falta de exploración de otras creencias. Finalmente, este estudio ha demostrado que existe bajo conocimiento en los padres de familia usuarios habituales del centro de salud Peralvillo-Huaral acerca de Epilepsia infantil, lo cual llama la atención sobre el rol educativo que tiene el sector salud. En consecuencia, 
se esperaría que los usuarios no habituales tengan un mayor grado de desconocimiento, siendo necesarios nuevos estudios de intervención educativa para mejorar el nivel de conocimiento en epilepsia infantil.

\section{AGRADECIMIENTO}

A la Dra. Roxana Ríos, médico del Centro de Salud de Peralvillo, por su apoyo en la realización de este estudio y a la población del Comité 16 del centro poblado de Peralvillo por su colaboración y activa participación en la encuesta y orientación educativa.

\section{Correspondencia:}

Melina Tejero Alcalde

Dirección: Calle Roma 335 - San Isidro

Teléfono: 956707 383/ 4221833

Correo electrónico: melina.tejero@upch.pe

\section{Conflicto de intereses:}

Ninguno

\section{REFERENCIAS BIBLIOGRÁFICAS}

1. Fisher RS, Van Emde Boas W, Blume W, Elger C, Genton P, Lee P, et al. Epileptic seizures and epi lepsy: definitions proposed by the international league against epilepsy (ILAE) and the international bureau for epilepsy (IBE). Epilepsia. 2005; 46: 470-2.

2. Medina C. Epilepsia: aspectos clínicos y psicosociles. Bogotá, Colombia: Editorial Médica Internacional Ltda; 2004. p. 39-40.

3. Burneo JG, Tellez-Zenteno J, Wiebe S. Understanding the burden of epilepsy in Latin America: A systematic review of its prevalence and incidence. Epilepsy Res. 2005;66:63-7)

4. Shaw EJ, Stokes T, Camosso-Stefinovic J, Baker R, Baker GA, Jacoby A. Educación en autocuidado para adultos con epilepsia. La Biblioteca Cochrane Plus: 2008;2. Disponible en: http://www.updatesoftware.com. ( Citado el 11 de enero del 2012).
5. Jarvie S, Colin A, Martin J. The development of a questionnaire to assess knowledge of epilepsy: 1general knowledge of epilepsy. Seizure. 1993; 2:179185.

6. Espinoza J, Madrid L, Soto F, Montoya M. Impacto de la información en epilepsia sobre las actitudes y creencias de un grupo de profesores. Rev Neuropsiquiatr.. 2002; 65: 104-112.

7. González AR, Fernández G, García R. Conocimientos y actitudes de las educadoras de círculos infantiles sobre epilepsia. Rev Cub Pediatr. 2010; 69(2): 118123.

8. Mendoza M, Miranda J, Guillén D, Samalvides F. Validación de una Encuesta para medir conocimientos y creencias sobre Epilepsia, en los padres de familia. Rev Med Hered. 2012; 23 (3): 160-165.

9. Hinnell C, Williams J, Metcalfe A, et al. Health status and health-related behaviors in epilepsy compared to other cronic conditions-A national populationbased study. Epilepsia. 2010;51:853-61.

10. Meyer AC, Dua T, Ma J, et al. Global disparities in the epilepsy treatment gap: A systematic review. Bull World Health Organ. 2010;88:250-6.

11. Saengsuwan J, et al. Factors associated with knowledge and attitudes in persons with epilepsy. Epilepsy Behav. 2012;24:23-9.

12. Tardillo L, Vila O, Guillén D. Conocimientos y Creencias Sobre Meningitis en Padres de Familia Residentes de las Localidades que Conforman la Micro - Red de Salud de Peralvillo - Huaral. Tesis para optar el Título de Médico Cirujano. Lima, Perú. Universidad Peruana Cayetano Heredia, 2009.

Recibido: 15/11/2012

Aceptado: 09/01/2013 


\section{ANEXO 1}

Universidad Peruana Cayetano Heredia

Facultad de Medicina Alberto Hurtado

Proyecto "Conocimientos y creencias acerca de Epilepsia en los padres de familia usuarios habituales del Centro de Salud de Peralvillo - Huaral"

Cuestionario final y respuestas correctas (las preguntas sobre creencias no tienen respuesta correcta)

\section{CUESTIONARIO}

1. Edad

2. Sexo

3. Estado civil

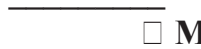

M $\quad \square \mathbf{F}$

4. Grado de instrucción $\square$ Primaria

$\square$ Conviviente $\square$ Soltero/a $\square$ Viudo/a

5. Religión

$\square$ Católica

Secundaria $\square$ Superior

6. Lugar de nacimiento: $\square$ Lima

$\square$ Evangélica

$\square$ Ninguna $\square$ Otra

7. Domicilio:

8. Tiempo de residencia en Lima:

9. ¿Sabe usted algo sobre Epilepsia?
$\square$ Sí
$\square$ No

Si contesto si, continúe la encuesta. Si contesto no, la encuesta ha concluido muchas gracias.

10. Marque ¿Cuál es la causa de la Epilepsia?

Trastorno de la función cerebral.

Enfermedad que se contagia

Castigo de Dios

Posesión maligna

Enfermedad que se hereda

11. Sabe usted ¿Quién debe tratar a la persona con Epilepsia?

Sacerdote

El señor de la farmacia

Médico

Chaman

Amigo

Si contesto médico pase a la siguiente; si no pase a la pregunta 13

Mendoza M, Miranda J, Guillén D, Samalvides F. Validación de una Encuesta para medir conocimientos y creencias sobre Epilepsia, 2009

12. Si contesto médico, ¿Qué médico especialista debe tratar a la persona que sufre de Epilepsia?

Endocrinólogo

Cardiólogo

Neurólogo

Cirujano

13. Sabe usted ¿A qué edad es más frecuente la Epilepsia?

Bebes (0-1)

Niños (1-9)

Adolescentes (9-18)

Ancianos (> 60)

Adultos (18 - 60) 
14. Una crisis convulsiva puede presentarse como:

Sacudidas del cuerpo

$\begin{array}{ll}\square \text { Sí } & \square \\ \square \text { Sí } & \square \text { No } \\ \square \text { Sí } & \square \text { No } \\ \square \text { Sí } & \square \text { No } \\ \square \text { Sí } & \square \text { No }\end{array}$

No Cambios en la expresión y miradas al vacío

Hablar solo al vacío

Visión de objetos inexistentes

$\square$ Sí $\quad \square$ No

15. Cree usted ¿Qué una persona que está teniendo una crisis epiléptica puede volverse agresiva y atacarlo?

$$
\square \text { Sí } \quad \square \text { No }
$$

16. Sabe usted ¿Qué se debe hacer ante una crisis convulsiva?

Poner a la persona de costado para que respire mejor

$\begin{array}{ll}\square \text { Sí } & \square \text { No } \\ \square \text { Sí } & \square \text { No } \\ \square \text { Sí } & \square \text { No } \\ \square \text { Sí } & \square \text { No } \\ \square \text { Sí } & \square \text { No } \\ \square \text { Sí } & \square \text { No } \\ \square \text { Sí } & \square \text { No } \\ \square \text { Sí } & \square \text { No }\end{array}$

Colocarle una almohada debajo de la cabeza

Colocar un pañuelo en la boca.

Sujetar al niño para impedir que siga sacudiéndose.

Tirarle agua encima para que reaccione

Aflojar o retirar corbatas, correas y/o collares

Ponerle un lápiz en la boca

Sobarle los brazos

$\square$ Sí $\quad \square$ No

17. ¿Qué factores pueden desencadenar una crisis epiléptica en una persona que sufre de Epilepsia?

Dormir pocas horas (menos de 6 horas)

$\begin{array}{ll}\square \text { Sí } & \square \text { No } \\ \square \text { Sí } & \square \text { No } \\ \square \text { Sí } & \square \text { No } \\ \square \text { Sí } & \square \text { No } \\ \square \text { Sí } & \square \text { No }\end{array}$

Dormir muy tarde

Tomar café

Tomar alcohol

$\square$ Sí $\square$ No

Mendoza M, Miranda J, Guillén D, Samalvides F. Validación de una Encuesta para medir conocimientos y creencias sobre Epilepsia

18. ¿Cuándo una crisis epiléptica puede dañar al cerebro?

Cuando el niño se sacude mucho tiempo

Cuando las crisis son muy frecuentes

$\square$ Sí $\quad \square$ No

Cuando se presenta fiebre

$\square$ Sí $\quad \square$ No

Cuando se presenta fiebre

$\square$ Sí $\square$ No

Cuando el niño vomita o saliva demasiado

$\square$ Sí $\square$ No

19. Sabe usted ¿Dónde debería atenderse una persona con Epilepsia para controlar su enfermedad?

En el hospital

En su propia casa

En la Iglesia

En la farmacia

En la casa de la vecina

20. ¿Cómo cree usted que se trata la Epilepsia?

Hierbas medicinales

Brebajes de sangre

Con rezos

Medicamentos

Con agua de azahar

Con baños de sanación

21. Sabe usted ¿Qué deportes pueden realizar los niños que sufren de Epilepsia?

Atletismo

Fútbol

Ping-pong

Juegos de mesa (ajedrez)

Buceo

$\square$ Sí $\quad \square$ No
$\square$ Sí $\quad \square$ No
$\square$ Sí $\quad \square$ No
$\square$ Sí $\quad \square$ No
$\square$ Sí $\quad \square$ No


22. ¿Qué actividades pueden realizar los niños que sufren de Epilepsia? Ir al colegio

Ir al cine

Estudiar

Sí $\square$ No

Bstudiar

Sí $\square$ No

Bailar

Sí $\square$ No

23. ¿Cree usted que las personas que sufren de epilepsia pueden ser útiles a su comunidad?

$\square$ Sí $\quad \square$ No

24. ¿Usted permitiría que un niño con epilepsia fuera amigo de su hijo/a?

$$
\square \text { Sí } \quad \square \text { No }
$$

Mendoza M, Miranda J, Guillén D, Samalvides F. Validación de una Encuesta para medir conocimientos y creencias sobre Epilepsia

25. ¿Cree usted que un niño con epilepsia puede ir a la misma escuela que un niño que no padece de Epilepsia?

$$
\square \text { Sí } \quad \square \text { No }
$$

26. ¿Cree usted que un niño con epilepsia es menos inteligente que un niño que no padece de Epilepsia?

27. ¿La epilepsia se hereda?

$\square$ Sí $\quad \square$ No

28. ¿La epilepsia se cura?

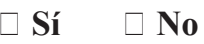

$\square$ Sí $\quad \square$ No

29. ¿A través de qué medio obtuvo la información que acerca de la Epilepsia?

De amigos, vecinos, familiares.

De campañas de salud en hospitales y postas

De un familiar o conocido que tiene Epilepsia

De la radio, periódico o televisión

Otro ¿cuál?

30. ¿Sabe usted si en el hospital se realizan charlas acerca de Epilepsia?

$\square$ Sí $\square$ No

31. Si contesto si, ¿Alguna vez ha asistido?

Mendoza M, Miranda J, Guillén D, Samalvides F. Validación de una Encuesta para medir conocimientos y creencias sobre Epi|lepsia 